\title{
PRINCIPALLY POLARIZABLE ISOGENY CLASSES OF ABELIAN SURFACES OVER FINITE FIELDS
}

\author{
Everett W. Howe, Daniel Maisner, Enric Nart, and Christophe \\ RITZENTHALER
}

\begin{abstract}
Let $\mathcal{A}$ be an isogeny class of abelian surfaces over $\mathbb{F}_{q}$ with Weil polynomial $x^{4}+a x^{3}+b x^{2}+a q x+q^{2}$. We show that $\mathcal{A}$ does not contain a surface that has a principal polarization if and only if $a^{2}-b=q$ and $b<0$ and all prime divisors of $b$ are congruent to 1 modulo 3 .
\end{abstract}

\section{Introduction}

An isogeny class of abelian varieties over a field $k$ is principally polarizable if it contains a variety that admits a principal polarization defined over $k$. If $k$ is algebraically closed then every isogeny class of abelian varieties over $k$ is principally polarizable, but over non-algebraically closed fields there may well exist isogeny classes that contain no principally polarized varieties. In general, it can be hard to tell whether an isogeny class of abelian varieties is principally polarizable. In this paper we consider the case of abelian surfaces over finite fields, and we give a simple criterion for deciding whether or not an isogeny class of such surfaces is principally polarizable. We use this result in a forthcoming paper [HNR06], in which we determine which isogeny classes of abelian surfaces contain Jacobians.

The Weil polynomial of an abelian variety over a finite field is the characteristic polynomial of its Frobenius endomorphism. The Honda-Tate theorem [Tat69] shows that two abelian varieties over a finite field are isogenous to one another if and only if they share the same Weil polynomial. If $A$ is an abelian surface over $\mathbb{F}_{q}$ then its Weil polynomial has the form

$$
x^{4}+a x^{3}+b x^{2}+a q x+q^{2}
$$

for some integers $a$ and $b$; suppressing the dependence on $q$, we will let $\mathcal{A}_{(a, b)}$ denote the isogeny class of the surface $A$.

Theorem 1. Let $\mathcal{A}=\mathcal{A}_{(a, b)}$ be an isogeny class of abelian surfaces over $\mathbb{F}_{q}$. Then $\mathcal{A}$ is not principally polarizable if and only if the following three conditions are satisfied:

(a) $a^{2}-b=q$,

(b) $b<0$, and

(c) all prime divisors of $b$ are congruent to 1 modulo 3 .

Received by the editors January 23, 2007.

2000 Mathematics Subject Classification. Primary 11G10; Secondary 11G25, 14G15.

Key words and phrases. abelian surface, Weil polynomial, principal polarization, finite field.

The third author was supported in part by project MTM2006-11391 from the Spanish MEC. 
Recall that an abelian surface over a field of characteristic $p$ is ordinary if the rank of its group of geometric $p$-torsion points is 2 ; it is supersingular if this rank is 0 . If an abelian surface over a field of positive characteristic is neither ordinary nor supersingular, we will call it mixed.

Several special cases of Theorem 1 appear in the literature or are easily derivable from known results. For simple ordinary abelian surfaces, Theorem 1 occurs as [How95, Thm. 1.3]. A straightforward argument using [MN02, Lem. 2.4] shows that the theorem holds for split isogeny classes. The second and third authors, using techniques from [How96], proved that every mixed isogeny class of abelian surfaces is principally polarizable [MN02, Thm. 4.3], and since an isogeny class $\mathcal{A}_{(a, b)}$ is mixed if and only if $p \nmid a$ and $p \mid b$ (so that $a^{2}-b \neq q$ ), Theorem 1 holds for these isogeny classes as well. For simple supersingular isogeny classes, Theorem 1 is equivalent to the following result.

Theorem 2. Let $\mathcal{A}$ be an isogeny class of simple supersingular abelian surfaces defined over $\mathbb{F}_{q}$, where $q$ is a power of a prime $p$. Then $\mathcal{A}$ is not principally polarizable if and only if $\mathcal{A}=\mathcal{A}_{(0,-q)}$ and $p \equiv 1 \bmod 3$.

To see that the two theorems are equivalent, we consult the complete list of simple supersingular isogeny classes given in [MN02, Thm. 2.9], and we note that the only class that meets the three conditions of Theorem 1 is the isogeny class mentioned in Theorem 2.

Correctly interpreted, Theorem 1 reveals a geometric feature of the isogeny classes that are not principally polarizable. Let $E$ be an elliptic curve over a finite field $k$ and let $\ell$ be the cubic extension of $k$. There is a natural trace map from the restriction of scalars $\operatorname{Res}(\ell / k, E)$ to $E$; the kernel $A$ of this map is the trace-zero surface associated to $E$. (The trace-zero surface is called the reduced restriction of scalars in [How01].) If the Weil polynomial for $E$ is $x^{2}-a x+q$ then $A$ lies in the isogeny class $\mathcal{A}_{\left(a, a^{2}-q\right)}$, so the isogeny class of $A$ satisfies the first condition of Theorem 1 . On the other hand, it is not hard to show that if an isogeny class $\mathcal{A}$ satisfies all three conditions of Theorem 1, then $\mathcal{A}$ contains the trace-zero surface of an elliptic curve over $\mathbb{F}_{q}$. (Indeed, for ordinary isogeny classes the first of the three conditions is sufficient.) These observations allow us to restate Theorem 1 as follows:

Theorem 3. Let $E$ be an elliptic curve defined over $\mathbb{F}_{q}$ and let a be the trace of Frobenius of E. Suppose that $|a|<\sqrt{q}$ and that all of the prime divisors of $a^{2}-$ $q$ are congruent to 1 modulo 3. Then the isogeny class of the trace-zero surface constructed from $E$ is not principally polarizable. Conversely, every isogeny class of abelian surfaces that is not principally polarizable contains the trace-zero surface of an elliptic curve whose trace satisfies the properties listed above.

In particular, we see that isogeny classes of abelian surfaces over finite fields that are not principally polarizable split over the cubic extension of the base field. The analogous statement for isogeny classes of higher dimension is false; for instance, there are absolutely simple isogeny classes of four-dimensional abelian varieties over $\mathbb{F}_{41}$ that are not principally polarizable [How95, Ex. 13.4].

The techniques we use to prove Theorem 1 are based on ideas from a series of papers [How95, How96, How01] in which the first author investigated the obstruction to the existence of a principally polarized variety in a given isogeny class over an 
arbitrary field. The general machinery provided in [How96] can in principle be used to determine whether or not an isogeny class of abelian varieties over a finite field is principally polarizable. In practice, however, there are certain situations in which one can apply the machinery only if one has sufficient information about some polarization of some variety in the isogeny class. One such situation arises in this paper.

In Section 2 we use results from [How96] to show that the simple supersingular isogeny classes not listed in Theorem 2 are principally polarizable. In Section 3 we prove Theorem 6, which specializes the results of [How96] to the case of certain simple isogeny classes and shows how the value of the Artin symbol of an ideal obtained from a polarization determines whether or not the variety is isogenous to a principally polarized variety. Finally, in Section 4 we use Theorem 6 to complete the proof of Theorem 2 by producing an explicit polarization of a trace-zero surface obtained from an elliptic curve and computing the Artin symbol of the associated ideal.

Notation. Let $k$ be a field and let $\ell$ be a finite extension of $k$. If $A$ is a variety over $k$ we let $A_{\ell}$ denote the variety $A \times \operatorname{Spec} k \operatorname{Spec} \ell$ over $\ell$. If $A$ is a variety over $\ell$, we let $\operatorname{Res}(\ell / k, A)$ denote the Weil restriction of scalars of $A$ from $\ell$ to $k$. Note that if $A$ is an abelian variety over $\ell$ that has a principal polarization, then $\operatorname{Res}(\ell / k, A)$ is an abelian variety over $k$ that has a principal polarization.

\section{Principally polarizable supersingular abelian surfaces}

In this section we prove the 'only if' direction of Theorem 2: We show that if $\mathcal{A}$ is an isogeny class of simple supersingular abelian surfaces over $\mathbb{F}_{q}$ of characteristic $p$, and if either $p \not \equiv 1 \bmod 3$ or $\mathcal{A} \neq \mathcal{A}_{(0,-q)}$, then $\mathcal{A}$ is principally polarizable. To prove this, we use one of the main theorems of [How96] together with a simple result. We start by proving the simple result.

Lemma 4. Let $A$ be a simple abelian surface defined over $\mathbb{F}_{q}$. Then $A_{\mathbb{F}_{q^{2}}}$ is not simple if and only if $A$ is isogenous to $\operatorname{Res}\left(\mathbb{F}_{q^{2}} / \mathbb{F}_{q}, E\right)$ for some elliptic curve $E$ defined over $\mathbb{F}_{q^{2}}$.

Proof. Suppose that $A \in \mathcal{A}_{(a, b)}$ and $A_{\mathbb{F}_{q^{2}}}$ is not simple. By [MN02, Thm.2.15] all simple mixed surfaces are absolutely simple; thus, $A$ is either ordinary or supersingular. By Lemma 2.1, Proposition 2.14, and Table 1 of [MN02], in both cases we have

- $a=0$, and

- $b$ is the trace of Frobenius of an elliptic curve $E$ over $\mathbb{F}_{q^{2}}$.

It follows from $[$ Mil72, $\S 1(\mathrm{a})]$ that the abelian surface $\operatorname{Res}\left(\mathbb{F}_{q^{2}} / \mathbb{F}_{q}, E\right)$ has the same Weil polynomial as does $A$, so the two surfaces are isogenous.

Conversely, if $A$ is isogenous to $\operatorname{Res}\left(\mathbb{F}_{q^{2}} / \mathbb{F}_{q}, E\right)$ then $A_{\mathbb{F}_{q^{2}}}$ is isogenous to the prod-



Remark. The Weil restriction of an elliptic curve is a principally polarizable variety, so the lemma shows that if $A_{\mathbb{F}_{q^{2}}}$ is not simple then $A$ is isogenous to a principally polarized variety.

Let us recall the first main theorem from [How96]. Suppose $\mathcal{A}$ is an isogeny class of simple abelian varieties over a finite field and let $f$ be its Weil polynomial. Since $\mathcal{A}$ is simple, the polynomial $f$ is a power of an irreducible polynomial. Let $\pi$ be a root of $f$ in $\mathbb{C}$. The field $K=\mathbb{Q}(\pi)$ is either totally real or a totally imaginary 


\begin{tabular}{|l|l|l|}
\hline$(a, b)$ & Conditions on $p, q$ & Reason for principal polarization \\
\hline \hline$(0,-q)$ & $p \equiv 1 \bmod 3, q$ nonsquare & - \\
\hline$(0,-q)$ & $p \equiv 7 \bmod 12, q$ square & - \\
\hline$(0,0)$ & $p \equiv 1 \bmod 4, q$ nonsquare & $K / K^{+}$ramified over 2 \\
\hline$(0,0)$ & $p \equiv 5 \bmod 8, q$ square & $K / K^{+}$ramified over 2 \\
\hline$(0, q)$ & $p \equiv 1 \bmod 3, q$ nonsquare & $K / K^{+}$ramified over 3 \\
\hline$( \pm \sqrt{q}, q)$ & $p \not \equiv 1 \bmod 5, q$ square & $K / K^{+}$ramified over 5 \\
\hline$( \pm \sqrt{5 q}, 3 q)$ & $p=5, q$ nonsquare & $K / K^{+}$ramified over 5 \\
\hline$( \pm \sqrt{2 q}, q)$ & $p=2, q$ nonsquare & $\pi-\bar{\pi}$ divisible by inert prime over 2 \\
\hline
\end{tabular}

TABLE 1. Certain irreducible supersingular Weil polynomials $x^{4}+$ $a x^{3}+b x^{2}+a q x+q^{2}$ over finite fields $\mathbb{F}_{q}$ of characteristic $p$, together with reasons (if any) why the corresponding isogeny class of abelian surfaces contains a principally polarized variety.

quadratic extension of a totally real field $K^{+}$. In either case, complex conjugation $x \mapsto \bar{x}$ induces an automorphism of $K$, trivial if $K$ is totally real.

Theorem 5 ([How96, Thm. 1.1]). If $K$ is totally real then $\mathcal{A}$ contains a principally polarized variety. Suppose $K$ is a CM-field. If a finite prime of $K^{+}$ramifies in $K / K^{+}$, or if there is a prime of $K^{+}$that divides $\pi-\bar{\pi}$ and that is inert in $K / K^{+}$, then $\mathcal{A}$ contains a principally polarized variety.

Now we have the machinery we need to prove the 'only if' direction of Theorem 2. Let $\mathcal{A}$ be an isogeny class of simple supersingular abelian surfaces over $\mathbb{F}_{q}$. If the surfaces in $\mathcal{A}$ split over $\mathbb{F}_{q^{2}}$ then Lemma 4 shows that there is a principally polarized surface in $\mathcal{A}$, so we need only concern ourselves with the isogeny classes that remain simple over $\mathbb{F}_{q^{2}}$. If the Weil polynomial of $\mathcal{A}$ is not irreducible, then the field $K$ has degree at most 2 over $\mathbb{Q}$, so either $K$ is totally real or $K$ is an imaginary quadratic extension of $\mathbb{Q}$ (and hence ramified at a finite prime). In either case, Theorem 5 shows that $\mathcal{A}$ is principally polarizable. Thus we need only consider isogeny classes whose Weil polynomials are irreducible.

Theorem 2.9 of [MN02], under the heading '(SS1)', lists the irreducible supersingular Weil polynomials coming from abelian surfaces. Table 1 of [MN02] lists the degrees of the smallest extension fields over which the isogeny classes split. Combining these sources, we can make a list of all of the Weil polynomials we must consider. We present this list in Table 1. For all but two of the entries, we give a reason why there is a principally polarized surface in the corresponding isogeny class.

We have shown that every isogeny class of simple supersingular abelian surfaces over $\mathbb{F}_{q}$ other than the first two entries of Table 1 is principally polarizable, which proves the 'only if' part of Theorem 2 .

Remark. At first glance it seems as if we have overlooked the case in which $q$ is a square, $p \equiv 1 \bmod 12$, and $\mathcal{A}=\mathcal{A}_{(0,-q)}$. But as we see from [MN02, Thm.2.9], there is no isogeny class with Weil polynomial $t^{4}-q t^{2}+q^{2}$ when $q$ is a square and $p \equiv 1 \bmod 12$. 


\section{Determining the existence of a principal polarization}

To complete the proof of Theorem 2, we must show that when $p \equiv 1$ mod 3 there is no principally polarized abelian surface with Weil polynomial $x^{4}-q x^{2}+q^{2}$. To accomplish this, we first provide a criterion for determining whether or not an isogeny class is principally polarizable; the criterion requires sufficient knowledge of an arbitrary polarization of a variety in the isogeny class, and is implicit in [How96]. Then, in Section 4, we construct a polarization of a variety in the isogeny class with Weil polynomial $x^{4}-q x^{2}+q^{2}$ to which we can apply the criterion.

Our criterion applies to isogeny classes of simple abelian varieties over $\mathbb{F}_{q}$ of arbitrary dimension. Let $\mathcal{A}$ be such an isogeny class, let $A$ be any variety in $\mathcal{A}$, and let $F$ and $V$ be the Frobenius and Verschiebung endomorphisms of $A$. Then the subring $R:=\mathbb{Z}[F, V]$ of $\operatorname{End}(A)$ is a domain, and up to isomorphism it is independent of the choice of $A \in \mathcal{A}$. Let $K:=\mathbb{Q}(F)$ be the quotient field of $R$. Then $K$ is either a totally real field or a CM-field, that is, a totally imaginary quadratic extension of a totally real field $K^{+}$.

As we noted above in Theorem 5 , if $K$ is totally real then $\mathcal{A}$ is principally polarizable; if $K$ is a CM-field that is ramified over $K^{+}$at a finite prime, then $\mathcal{A}$ is principally polarizable; and if $F-V$ is divisible by a prime of $K^{+}$that is inert in $K / K^{+}$, then $\mathcal{A}$ is principally polarizable. Thus, we may henceforth assume that $K$ is a CM-field that is unramified over $K^{+}$at every finite prime, and that every prime of $K^{+}$that divides $F-V$ is split in $K / K^{+}$.

Let $\mathcal{O}$ be the ring of integers of $K$ and let $\mathcal{O}^{+}$be the ring of integers of $K^{+}$. Let $A$ be any variety in $\mathcal{A}$ such that $\mathcal{O} \subseteq \operatorname{End}(A)$; we know from [Wat69, Thm. 3.13] that such varieties exist. Let $\widehat{A}$ be the dual variety of $A$, and suppose there is a polarization $\lambda: A \rightarrow \widehat{A}$ of $A$ whose degree is coprime to the characteristic of $k$. Then the group of geometric points of $\operatorname{ker}(\lambda)$ is naturally an $\mathcal{O}$-module, and the proof of [How96, Prop. 7.1] shows that there is an ideal $\mathfrak{A}$ of $\mathcal{O}^{+}$such that

$$
\operatorname{ker}(\lambda) \cong \mathcal{O} / \mathfrak{A O}
$$

as $\mathcal{O}$-modules. Let $\psi$ denote the Artin map from the ideal group of $\mathcal{O}^{+}$to the Galois group of $K$ over $K^{+}$; we identify this Galois group with $\{ \pm 1\}$.

Theorem 6. The isogeny class $\mathcal{A}$ is principally polarizable if and only if $\psi(\mathfrak{A})=1$.

Proof. In [How96, §6] the first author defined a contravariant functor $\mathcal{B}$ from a certain category of rings (the "proper real/CM-orders") to the category of finite 2-torsion groups. In particular, for every order $S$ in $K$ that is fixed by complex conjugation, we get a group $\mathcal{B}(S)$, and the inclusion map $i: R \rightarrow \mathcal{O}$ gives us a group homomorphism

$$
i^{*}: \mathcal{B}(\mathcal{O}) \rightarrow \mathcal{B}(R) .
$$

Furthermore, [How96, Prop. 6.2] shows that

$$
\mathcal{B}(\mathcal{O}) \cong \mathrm{Cl}^{+}\left(\mathcal{O}^{+}\right) / N_{\mathcal{O} / \mathcal{O}^{+}}(\mathrm{Cl}(\mathcal{O}))
$$

so that the Artin map gives an isomorphism from $\mathcal{B}(\mathcal{O})$ to $\operatorname{Gal}\left(K / K^{+}\right)$.

The third main result of [How96] (Theorem 1.3) says that there is a naturallydefined element $I_{\mathcal{A}}$ of $\mathcal{B}(R)$ that is zero precisely when $\mathcal{A}$ is principally polarizable. Proposition 7.1 of [How96] shows that $I_{\mathcal{A}}$ lies in the image of $\mathcal{B}(\mathcal{O})$ under $i^{*}$. In particular, the proof of [How96, Prop. 7.1] shows that $I_{\mathcal{A}}$ is the image under $i^{*}$ of the 
class of the ideal $\mathfrak{A}$. Proposition 7.2 of [How96] says that under our assumptions on $A$ (namely, that $K$ is a CM-field that is unramified over $K^{+}$at all finite primes, and that no inert prime of $K^{+}$divides $F-V$ ), the map $i^{*}$ is an injection. Therefore, $I_{\mathcal{A}}$ is zero if and only if the class of $\mathfrak{A}$ in $\mathcal{B}(\mathcal{O})$ is zero, and this happens if and only if the Artin symbol on $\mathfrak{A}$ is trivial.

Remark. It is only for convenience that we assume that the polarization $\lambda$ of $A$ has degree coprime to the characteristic. We can associate an ideal $\mathfrak{A}$ of $\mathcal{O}$ to any polarization of $A$ as in the proof of [How96, Prop. 7.1], and Theorem 6 remains true for the ideals associated to these more general polarizations.

\section{An explicit polarization}

To use Theorem 6 to complete the proof of Theorem 2, we must construct an explicit polarization of an abelian surface in the isogeny class with Weil polynomial $x^{4}-q x^{2}+q^{2}$. In this section we construct such a polarization by using ideas from [How01]. We assume throughout this section that $p \equiv 1 \bmod 3$ and that $p \equiv 3 \bmod 4$ if $q$ is a square.

Our assumptions on $p$ and $q$ guarantee that there is a supersingular elliptic curve $E$ over $\mathbb{F}_{q}$ with Weil polynomial $x^{2}+q$ (see [Wat69, Thm. 4.1]). Furthermore, by [Wat69, Thm. 3.13] we can choose $E$ so that its endomorphism ring is the maximal order $S$ of the field $L:=\mathbb{Q}(\sqrt{-q})$.

Let $A$ be the trace-zero surface over $k$ associated to $E$, by which we mean the kernel of the trace map from $\operatorname{Res}\left(\mathbb{F}_{q^{3}} / \mathbb{F}_{q}, E_{\mathbb{F}_{q^{3}}}\right)$ to $E$. By [Mil72, $\left.\S 1(\mathrm{a})\right]$, the Weil polynomial of $A$ is $f=x^{4}-q x^{2}+q^{2}$, so $A$ lies in the isogeny class $\mathcal{A}=\mathcal{A}_{(0,-q)}$ that we are interested in. Let $F$ and $V$ be the Frobenius and Verschiebung endomorphisms of $A$; since $f$ is irreducible, the subring $K=\mathbb{Q}(F)$ of $\operatorname{End}(A) \otimes \mathbb{Q}$ is a field. In fact, $K$ is a CM-field whose maximal real subfield $K^{+}$is isomorphic to $\mathbb{Q}(\sqrt{3 q})$. There are also two imaginary quadratic subfields of $K$; one of them is isomorphic to the endomorphism algebra $L=\mathbb{Q}(\sqrt{-q})$ of $E$, and one is $M:=\mathbb{Q}(\omega)$, where $\omega$ is a primitive cube root of unity.

The discriminants of $L$ and $M$ are coprime to each other, so the field $K$ is unramified over $K^{+}$at all finite primes. We compute that $(F-V)^{2}=-q$, that there is a unique prime $\mathfrak{p}$ of $K^{+}$over $p$, and that $\mathfrak{p}$ splits in $K$. Thus the field $K$ satisfies all of the hypotheses set forth at the beginning of Section 3.

In Section 2.2 of [How01] it is shown that $A$ is the $\mathbb{F}_{q^{3}} / \mathbb{F}_{q^{-}}$-twist of $E \times E$ corresponding to the element of $H^{1}\left(\mathrm{Gal}\left(\mathbb{F}_{q^{3}} / \mathbb{F}_{q}\right)\right.$, Aut $\left.(E \times E)\right)$ represented by the cocyle that sends the Frobenius automorphism of $\mathbb{F}_{q^{3}} / \mathbb{F}_{q}$ to the automorphism of $E \times E$ given by the matrix

$$
\zeta=\left[\begin{array}{cc}
-1 & -1 \\
1 & 0
\end{array}\right]
$$

By replacing $\omega$ with its complex conjugate, if necessary, we may assume that $\zeta=\omega$. Note that every geometric endomorphism of $E \times E$ is defined over $\mathbb{F}_{q}$, and that the endomorphism ring of $E \times E$ is isomorphic to the ring $M_{2}(S)$ of $2 \times 2$ matrices over $S$. Proposition 2.1 of [How01] says that an element of $M_{2}(S)$ gives rise to an endomorphism of $A$ if and only if it commutes with the element $\zeta$. This shows that End $A \cong S[\omega]$. Since the discriminants of $L$ and $M$ are coprime to one another, the 
ring $S[\omega]$ is the full ring of integers $\mathcal{O}$ of $K$. Thus we can apply Theorem 6 to the variety $A$ if we can find an explicit polarization of $A$.

Let $b$ be the endomorphism

$$
\left[\begin{array}{ll}
2 & 1 \\
1 & 2
\end{array}\right]
$$

of $E \times E$, let $\mu$ be the product polarization of $E \times E$, and let $\lambda=\mu b$, so that $\lambda$ is a polarization of degree 9. As in the proof of [How01, Lem. 2.6], Proposition 2.2 of [How01] shows that $\lambda$ gives rise to a polarization on $A$. As we noted at the beginning of Section 3, there is an ideal $\mathfrak{A}$ of the ring of integers $\mathcal{O}^{+}$of $K^{+}$such that $\operatorname{ker}(\lambda) \cong \mathcal{O} / \mathfrak{A O}$, and the only possibility is that $\mathfrak{A}$ is the unique prime of $\mathcal{O}^{+}$lying over the rational prime 3 .

The prime 3 ramifies in $M / \mathbb{Q}$ and is inert in $L / \mathbb{Q}$, so the prime $\mathfrak{A}$ is inert in $K / K^{+}$. Therefore its Artin symbol is nontrivial, and it follows from Theorem 6 that the isogeny class $\mathcal{A}$ is not principally polarizable. This completes the proof of Theorem 2 .

\section{References}

[How95] Everett W. Howe: Principally polarized ordinary abelian varieties over finite fields, Trans. Amer. Math. Soc. 347 (1995) 2361-2401.

[How96] Everett W. Howe: Kernels of polarizations of abelian varieties over finite fields, J. Algebraic Geom. 5 (1996) 583-608.

[How01] Everett W. Howe: Isogeny classes of abelian varieties with no principal polarizations, pp. 203-216 in: Moduli of abelian varieties (C. Faber, G. van der Geer and F. Oort, eds.), Progr. Math. 195, Birkhäuser, Basel, 2001.

[HNR06] Everett W. Howe, Enric Nart, and Christophe Ritzenthaler: Jacobians in isogeny classes of abelian surfaces over finite fields, in preparation.

[Mn02] Daniel Maisner and Enric Nart with an appendix by Everett W. Howe: Abelian surfaces over finite fields as Jacobians, Experiment. Math. 11 (2002) 321-337.

[Mil72] J. S. MiLne: On the arithmetic of abelian varieties, Invent. Math. 17 (1972) 177-190.

[Tat69] John Tate: Classes d'isogénie des variétés abéliennes sur un corps fini (d'après T. Honda), Exp. No. 352, pp. 95-110 in: Séminaire Bourbaki 1968/69, Lecture Notes in Math. 179, Springer-Verlag, Berlin 1971.

[Wat69] William C. Waterhouse: Abelian varieties over finite fields, Ann. Sci. École Norm. Sup. (4) 2 (1969) 521-560.

Center for Communications Research, 4320 Westerra Court, San Diego, CA 92121-1967, USA.

E-mail address: however@alumni.caltech.edu

$U R L:$ http://www.alumni.caltech.edu/ however/

Universidad Autónoma de la Ciudad de México (UACM), Plantel San lorenzo Tezonco, Colegio de Ciencia y Tecnología, Prolongación San Isidro \# 151, San Lorenzo Tezonco, CP 09790 IzTapalapa, D.F. MÉXico.

E-mail address: danielm@mat.uab.es

Departament de Matemàtiques, Universitat Autònoma de Barcelona, Edifici C, 08193 Bellaterra, Barcelona, Spain.

E-mail address: nart@mat.uab.es

Institut de Mathématiques de Luminy, UMR 6206 du CNRS, Luminy, Case 907, 13288 Marseille, France.

E-mail address: ritzenth@iml.univ-mrs.fr 